\title{
Hiperpigmentação em mucosa oral de pacientes usuários de cloroquina e
}

\section{hidroxicloroquina: Revisão de literatura}

Hyperpigmentation in the oral mucosa of patients using chloroquine and hydroxychloroquine: A literature review

Hiperpigmentación en la mucosa oral de pacientes que utilizan cloroquina e hidroxicloroquina:

Revisión de la literatura

Júlia Kiara da Nóbrega Holanda ORCID: https://orcid.org/0000-0002-1426-0714 Universidade Federal de Campina Grande, Brasil E-mail: juliakiaranh@gmail.com

Lara Danúbia Galvão de Souza ORCID: https://orcid.org/0000-0001-5693-9453 Universidade Federal de Campina Grande, Brasil E-mail: lara.danubia@outlook.com

Lorena Layanne Pereira Custódio ORCID: https://orcid.org/0000-0002-6379-5993 Universidade Federal de Campina Grande, Brasil E-mail: lorenalayanne888@gmail.com

Maria Luiza Dantas da Silva ORCID: https://orcid.org/0000-0001-6694-8388 Universidade Federal de Campina Grande, Brasil

E-mail: luizadantasodontologia@gmail.com

Aliny Thaisy Araújo Costa

ORCID: https://orcid.org/0000-0002-9120-7386

Universidade Federal de Campina Grande, Brasil E-mail: thaisyaliny@gmail.com

Debora Lana Alves Monteiro ORCID: https://orcid.org/0000-0002-4731-6737

Universidade Estadual da Paraíba, Brasil E-mail: a.deboralana@gmail.com

Rafaela Oliveira Santos ORCID: https://orcid.org/0000-0002-3072-8874 Universidade Federal de Campina Grande, Brasil

E-mail: rafaela_ob@hotmail.com

Igor Natanyo de Freitas Silva ORCID: https://orcid.org/0000-0001-8996-7370 Universidade Federal de Campina Grande, Brasil E-mail: igornatanyo2@gmail.com

Alexandre José da Costa ORCID: https://orcid.org/0000-0002-4014-8689 Universidade Federal de Campina Grande, Brasil

E-mail: alexandre.jose_costa@hotmail.com Gabrielli Bezerra Sales

ORCID: https://orcid.org/0000-0003-1842-9844 Universidade Federal de Campina Grande, Brasil E-mail: gabrielli.b.sales@ gmail.com Janaina Barbosa de Alencar ORCID: https://orcid.org/0000-0003-4270-4375 Universidade Federal de Campina Grande, Brasil E-mail: ja.naina.b@hotmail.com

Sérvulo da Costa Rodrigues Neto ORCID: https://orcid.org/0000-0002-3823-5759 Universidade Federal de Campina Grande, Brasil

E-mail: servulorodriguesneto@gmail.com

João Nilton Lopes de Sousa ORCID: https://orcid.org/0000-0003-3726-386X Universidade Federal de Campina Grande, Brasil E-mail: jnlopesodonto@gmail.com 


\begin{abstract}
Resumo
A nova classificação das doenças e condições periodontais e peri-implantares publicada em 2018 inclui as patologias e doenças gengivais não induzidas por biofilme, incluindo as pigmentações gengivais relacionadas a medicamentos. A cloroquina e hidroxicloroquina são usados há anos devido seus efeitos imunossupressores e anti-inflamatórios e agora são alvos de estudos sobre possível eficácia contra o novo coronavírus (SARS-CoV-2). Este estudo tem como objetivo identificar os relatos de casos de pigmentação oral relacionados ao uso crônico de cloroquina e hidroxicloroquina. Foi realizado um levantamento de artigos científicos, utilizando como descritores os termos hiperpigmentação, cloroquina, hidroxicloroquina, nas bases de dados: Google acadêmico, SciElo, PubMed, Medline e LILACS. Foram selecionados 12 estudos do tipo relato de caso e série de casos com 18 casos clínicos enquadrados na temática deste estudo. Esses foram avaliados levando em consideração as características da hiperpigmentação, o medicamento usado, motivo e tempo do uso, além da idade e sexo do paciente. Dos 18 casos analisados, 15 encontravam-se no palato duro. Além disso, as pigmentações acometeram mais o sexo feminino e apresentavam coloração cinza-azulado ou pretoazulado. Portanto, é crucial que o cirurgião-dentista esteja atento para realizar o diagnóstico diferencial com outras lesões que apresentam potencial de malignidade.
\end{abstract}

Palavras-chave: Hiperpigmentação; Cloroquina; Hidroxicloroquina.

\begin{abstract}
The new classification of periodontal and peri-implant diseases and conditions published in 2018 includes gum pathologies and diseases not induced by biofilm, including drug-related gingival pigmentations. Chloroquine and hydroxychloroquine have been used for years due to their immunosuppressive and anti-inflammatory effects and are now the target of studies on possible efficacy against the new coronavirus (SARS-CoV-2). This study aims to identify the case reports of oral pigmentation related to chronic use of chloroquine and hydroxychloroquine. A survey of scientific articles was carried out, using the terms hyperpigmentation, chloroquine, hydrox ychloroquine as descriptors, in the databases: Google scholar, SciElo, PubMed, Medline and LILACS. Twelve case report and case series studies were selected with 18 clinical cases within the scope of this study. These were evaluated taking into account the characteristics of hyperpigmentation, medication used, reason and time of use, in addition to the patient's age and sex. Of the 18 cases analyzed, 15 were found on the hard palate. In addition, pigmentations were more prevalent in females and had a bluish-gray or bluish-black color. Therefore, it is crucial that the dental surgeon is attentive to make the differential diagnosis with other lesions that have the potential for malignancy.
\end{abstract}

Keywords: Hyperpigmentation; Chloroquine; Hydroxychloroquine.

\title{
Resumen
}

La nueva clasificación de enfermedades y afecciones periodontales y periimplantarias publicada en 2018 incluye patologías de las encías y enfermedades no inducidas por biopelículas, incluidas las pigmentaciones gingivales relacionadas con fármacos. La cloroquina y la hidroxicloroquina se han utilizado durante años debido a sus efectos inmunosupresores y antiinflamatorios y ahora son el objetivo de estudios sobre la posible eficacia contra el nuevo coronavirus (SARS-CoV-2). Este estudio tiene como objetivo identificar los informes de casos de pigmentación oral relacionados con el uso crónico de cloroquina e hidroxicloroquina. Se realizó un relevamiento de artículos científicos, utilizando como descriptores los términos hiperpigmentación, cloroquina, hidroxicloroquina, en las bases de datos: Google Scholar, SciElo, PubMed, Medline y LILACS. Se seleccionaron 12 informes de casos y estudios de series de casos con 18 casos clínicos dentro del alcance de este estudio. Estos se evaluaron teniendo en cuenta las características de la hiperpigmentación, la medicación utilizada, el motivo y el momento de uso, además de la edad y el sexo del paciente. De los 18 casos analizados, 15 se encontraron en el paladar duro. Además, las pigmentaciones eran más frecuentes en las hembras y tenían un color gris azulado o negro azulado. Por tanto, es fundamental que el cirujano dentista esté atento para realizar el diagnóstico diferencial con otras lesiones que tienen potencial de malignidad.

Palabras clave: Hiperpigmentación; Cloroquina; Hidroxicloroquina.

\section{Introdução}

Um sorriso harmônico é um elemento importante para a autoestima de um indivíduo. Para que isso seja possível, a cor, forma e posição do dente devem estar em harmonia com o tecido gengival. Já a coloração gengival está relacionada principalmente com fatores como: a quantidade de vascularização da área, espessura do epitélio, grau de queratinização e a existência de pigmentos dentro do epitélio gengival (Shahna, Suchetha, Sapna, Darshan \& Apoorva, 2009).

Alguns fatores como trauma, hormônios, radiação e medicamentos podem levar a estimulação da produção de melanina, podendo resultar em pigmentações gengivais (Gulati, Dutt, Gupta \& Tyagi, 2016). Sendo assim, as pigmentações dos tecidos moles na cavidade bucal necessitam de uma atenção especial, uma vez que existem inúmeras alterações 
relacionadas a essa condição, podendo surgir dificuldades para um correto diagnóstico devido às suas diferentes origens e formas de apresentação clínica (Holmstrup, Plemons \& Meyle, 2018).

A mais nova classificação das doenças e condições periodontais e peri-implantares publicada em 2018 inclui as patologias e doenças gengivais não induzidas por biofilme. Elas são, em sua grande maioria, manifestações de condições sistêmicas, mas também podem representar alterações patológicas limitadas aos tecidos do periodonto (Steffens \& Marcantonio, 2018). Dentre as patologias e doenças gengivais não induzidas pelo biofilme estão às pigmentações gengivais, que podem estar relacionadas a medicamentos. A hiperpigmentação induzida por terapêutica medicamentosa pode ser causada pela produção aumentada de melanina com ou sem aumento de melanócitos, depósito dérmico da droga ou de seus metabólitos, produção de pigmentos sob a influência da droga, ou provenientes da destruição das células vermelhas do sangue (Sreeja et al., 2015).

Entre os medicamentos que podem ser causadores de mudanças de coloração da mucosa bucal estão os laxantes (fenolftaleína), antibióticos (minociclina), tranquilizantes (clorpromazina), antimaláricos, agentes quimioterápicos (doxorrubicina, bussulfano e ciclofosfamida), estrogênio e medicamentos antirretrovirais (AZT e cetoconazol) (de Andrade et al., 2013; Godinho, Paz, Gomes, Garcia \& Volpato, 2020).

A cloroquina e a hidroxicloroquina são classificadas como agentes antimaláricos por seus efeitos imunossupressores e anti-inflamatórios, e podem promover na mucosa o aparecimento de pigmentação difusa enegrecida ou azul-acinzentada, a qual ocorre mais frequentemente no palato duro (de Andrade et al., 2013; de Andrade, Padron-Alvarado, Muñoz-Campos, Morais \& Martinez-Pedraza, 2017; Tosios, Kalogirou \& Sklavounou, 2018). Estudos recentes evidenciam que tanto a cloroquina quanto a hidroxicloroquina têm possível eficácia no tratamento de pacientes infectados pelo novo coronavírus (SARS-CoV-2) que estejam em quadro clínico moderado ou grave (Wang et al., 2020). A epidemia, que começou em dezembro de 2019 em Wuhan (província de Hubei, China), foi declarada uma pandemia pela Organização Mundial da Saúde (OMS), com substancial morbimortalidade relatada em vários países, sem que haja até agora uma vacina ou tratamento médico comprovado para a doença (COVID-19) (Dong, Hu \& Gao, 2020; Gao, Tian \& Yang, 2020; Xueting et al., 2020).

Portanto, espera-se que o uso de tais medicações tenha um aumento considerável, e consequentemente, torna-se importante para os cirurgiões-dentistas e demais profissionais da área reconhecerem seus possíveis efeitos colaterais e se atualizarem sobre como essa pandemia está relacionada à sua profissão, a fim de estarem bem orientados e preparados. Assim, este estudo tem como objetivo identificar, na literatura, os relatos de casos de pigmentação oral relacionados ao uso crônico de cloroquina e hidroxicloroquina, além de abordar o contexto atual auxiliando profissionais da área da Odontologia em sua identificação e diagnóstico diferencial.

\section{Metodologia}

O artigo constituiu-se de levantamento de casos de hiperpigmentação de mucosa oral causada pelo uso crônico de cloroquina ou hidroxicloroquina por pacientes em tratamento de alguma condição sistêmica, publicadas nas bases de dados de artigos científicos Google acadêmico, SciElo, PubMed, Medline e LILACS, utilizando os termos hiperpigmentação/ hyperpigmentation, cloroquina/chloroquine, hidroxicloroquina/ hydroxychloroquine.

De acordo com Pereira, Shitsuka, D. M., Parreira, e Shitsuka, R (2018), as revisões de literatura devem ser aprofundadas e detalhadas de forma a evitar trabalhos superficiais que refletem a opinião de poucos autores e que possam ser tendenciosas. Dessa forma, os artigos incluídos neste estudo foram aqueles de língua portuguesa e inglesa que trataram de relato de caso ou série de casos de relevância da temática supracitada, sem delimitação de tempo.

Assim, no sistema de busca deste trabalho, foram encontrados 12 artigos, contendo 18 casos de hiperpigmentação de mucosa oral pelo uso crônico de cloroquina ou hidroxicloroquina. Com isso, buscou-se analisar nos casos, essencialmente, 
idade e sexo do paciente, a região de acometimento na mucosa oral, as características clínicas da hiperpigmentação, qual medicamento utilizado pelo paciente (cloroquina ou hidroxicloroquina), motivo e tempo do uso.

\section{Revisão da Literatura}

De acordo com a metodologia empregada, foram selecionados 18 casos de hiperpigmentação da mucosa oral causada pelo uso crônico de cloroquina e/ou hidroxicloroquina (Quadro 1).

Quadro 1. Casos de hiperpigmentação oral pelo uso crônico de cloroquina e hidroxicloriquina relatados na literatura.

\begin{tabular}{|c|c|c|c|c|c|}
\hline Tipo de estudo & $\begin{array}{l}\text { Idade } \\
\text { / sexo }\end{array}$ & $\begin{array}{l}\text { Região da } \\
\text { mucosa oral }\end{array}$ & $\begin{array}{c}\text { Descrição da } \\
\text { hiperpigmentação }\end{array}$ & $\begin{array}{l}\text { Medicamento / } \\
\text { tempo de uso }\end{array}$ & $\begin{array}{l}\text { Motivo do } \\
\text { uso }\end{array}$ \\
\hline $\begin{array}{c}\text { Série de casos } \\
\text { (Tosios et al., 2018) }\end{array}$ & $53 / \mathrm{F}$ & $\begin{array}{l}\text { Palato duro e } \\
\text { gengiva } \\
\text { inserida do } \\
\text { elemento } 33\end{array}$ & $\begin{array}{l}\text { Mancha azul-amarronzada } \\
\text { homogênea e difusa, não } \\
\text { desapareceu à pressão }\end{array}$ & $\begin{array}{c}\text { Hidroxicloroquina } 400 \\
\text { mg/dia / } \\
5 \text { anos }\end{array}$ & $\begin{array}{l}\text { Artrite } \\
\text { reumatoide }\end{array}$ \\
\hline $\begin{array}{c}\text { Relato de caso } \\
\text { (Godinho et al., 2020) }\end{array}$ & $66 / \mathrm{F}$ & Palato duro & $\begin{array}{c}\text { Mancha preta-azulada, mal } \\
\text { delimitada, com cerca de } 3 \\
\text { cm de diâmetro }\end{array}$ & $\begin{array}{c}\text { Cloroquina } \\
250 \mathrm{mg} / \mathrm{dia} / \text { paciente } \\
\text { não recordava }\end{array}$ & $\begin{array}{l}\text { Artrite } \\
\text { reumatoide }\end{array}$ \\
\hline $\begin{array}{l}\text { Relato de caso } \\
\text { (Andrade, Rojas, } \\
\text { Rodríguez \& } \\
\text { Vázquez, 2019) }\end{array}$ & $58 / \mathrm{F}$ & Língua & $\begin{array}{l}\text { Manchas difusas azul } \\
\text { acinzentadas }\end{array}$ & $\begin{array}{l}\text { Hidroxicloroquina } 200 \\
\text { mg/dia / } 4 \text { meses }\end{array}$ & $\begin{array}{l}\text { Lúpus } \\
\text { eritematoso } \\
\text { sistêmico }\end{array}$ \\
\hline $\begin{array}{c}\text { Relato de caso } \\
\text { (Attuati, Martini, } \\
\text { Bonacina, Mariani \& } \\
\text { Gianatti, 2019) }\end{array}$ & $87 / M$ & Palato duro & $\begin{array}{c}\text { Mancha cinza, homogênea, } \\
\text { assintomática, simétrica e } \\
\text { bilateral }\end{array}$ & $\begin{array}{l}\text { Cloroquina } 250 \\
\text { mg/dia / não } \\
\text { informado }\end{array}$ & $\begin{array}{l}\text { Artrite } \\
\text { reumatoide }\end{array}$ \\
\hline $\begin{array}{c}\text { Relato de caso } \\
\text { (Horta-Baas, 2018) }\end{array}$ & $61 / \mathrm{F}$ & $\begin{array}{l}\text { Lábios e } \\
\text { mucosa jugal }\end{array}$ & $\begin{array}{l}\text { Manchas enegrecidas com } \\
\text { bordas bem definidas }\end{array}$ & $\begin{array}{l}\text { Cloroquina } 150 \\
\mathrm{mg} / \text { dia / } 2 \text { anos }\end{array}$ & $\begin{array}{l}\text { Lúpus } \\
\text { eritematoso } \\
\text { sistêmico }\end{array}$ \\
\hline $\begin{array}{l}\text { Relato de caso } \\
\text { (Kalampalikis, } \\
\text { Goetze \& Elsner, } \\
\text { 2012) }\end{array}$ & $55 / \mathrm{F}$ & $\begin{array}{c}\text { Gengiva } \\
\text { anterior inferior }\end{array}$ & Mancha azul-acinzentada & $\begin{array}{l}\text { Hidroxicloroquina } 200 \\
\mathrm{mg} / \mathrm{dia} / \mathrm{l} \text { ano }\end{array}$ & $\begin{array}{l}\text { Síndrome de } \\
\text { Sjögren }\end{array}$ \\
\hline \multirow{2}{*}{$\begin{array}{l}\text { Relato de caso } \\
\text { (da Silveira, Quadros, } \\
\text { Maito \& Giordani, } \\
\text { 2003) }\end{array}$} & $56 / \mathrm{F}$ & Palato duro & Mancha negro-azulada & $\begin{array}{l}\text { Cloroquina } 500 \\
\text { mg/dia / não } \\
\text { informado }\end{array}$ & $\begin{array}{l}\text { Lúpus } \\
\text { eritematoso } \\
\text { discoide }\end{array}$ \\
\hline & $40 / \mathrm{F}$ & Palato duro & Mancha cinza-azulada de & Cloroquina (dose não & Lúpus \\
\hline
\end{tabular}


Research, Society and Development, v. 10, n. 5, e30010514528, 2021

(CC BY 4.0) | ISSN 2525-3409 | DOI: http://dx.doi.org/10.33448/rsd-v10i5.14528

\begin{tabular}{|c|c|c|c|c|c|}
\hline & & & $\begin{array}{l}\text { grande extensão e bem } \\
\text { delimitada }\end{array}$ & informada) / 1 ano & $\begin{array}{l}\text { eritematoso } \\
\text { discoide }\end{array}$ \\
\hline \multirow{2}{*}{$\begin{array}{c}\text { Relato de caso } \\
\text { (Gallo, Luiz, } \\
\text { Ferrazzo, Migliari \& } \\
\text { Sugaya, 2009) }\end{array}$} & $65 / \mathrm{F}$ & Palato duro & $\begin{array}{l}\text { Mancha difusa azul- } \\
\text { acinzentada }\end{array}$ & $\begin{array}{l}\text { Cloroquina (dose não } \\
\text { informada) / } 3 \text { anos }\end{array}$ & $\begin{array}{l}\text { Artrite } \\
\text { reumatoide }\end{array}$ \\
\hline & $81 / \mathrm{F}$ & Palato duro & $\begin{array}{l}\text { Mancha difusa azul- } \\
\text { acinzentada }\end{array}$ & $\begin{array}{l}\text { Cloroquina (dose não } \\
\text { informada) / } 10 \text { anos }\end{array}$ & $\begin{array}{l}\text { Síndrome de } \\
\text { Sjögren }\end{array}$ \\
\hline \multirow{5}{*}{$\begin{array}{l}\text { Série de casos } \\
\text { (de Andrade et al., } \\
\text { 2013) }\end{array}$} & $54 / \mathrm{F}$ & Palato duro & $\begin{array}{c}\text { Mancha azul-acinzentada } \\
\text { difusa, de } 5 \mathrm{~cm} \text {, com bordas } \\
\text { irregulares }\end{array}$ & $\begin{array}{l}\text { Cloroquina } 250 \\
\text { mg/dia / } 4 \text { anos }\end{array}$ & $\begin{array}{l}\text { Artrite } \\
\text { reumatoide }\end{array}$ \\
\hline & $83 / \mathrm{F}$ & Palato duro & $\begin{array}{l}\text { Mancha difusa azul- } \\
\text { acinzentada }\end{array}$ & $\begin{array}{l}\text { Cloroquina } 250 \\
\mathrm{mg} / \mathrm{dia} / 3 \text { anos }\end{array}$ & $\begin{array}{l}\text { Artrite } \\
\text { reumatoide }\end{array}$ \\
\hline & $60 / M$ & Palato duro & Mancha escura e indolor & $\begin{array}{l}\text { Cloroquina } 250 \\
\text { mg/dia / } 12 \text { anos }\end{array}$ & $\begin{array}{l}\text { Artrite } \\
\text { reumatoide }\end{array}$ \\
\hline & $59 / \mathrm{M}$ & Palato duro & $\begin{array}{l}\text { Mancha azul-acinzentada } \\
\text { difusa e indolor }\end{array}$ & $\begin{array}{l}\text { Cloroquina } 250 \\
\mathrm{mg} / \mathrm{dia} / 10 \text { anos }\end{array}$ & $\begin{array}{l}\text { Artrite } \\
\text { reumatoide }\end{array}$ \\
\hline & $65 / F$ & Palato duro & $\begin{array}{l}\text { Mancha azul-acinzentada } \\
\text { difusa e assintomática }\end{array}$ & $\begin{array}{l}\text { Cloroquina } 250 \\
\mathrm{mg} / \mathrm{dia} / 3 \text { anos }\end{array}$ & $\begin{array}{l}\text { Artrite } \\
\text { reumatoide }\end{array}$ \\
\hline $\begin{array}{c}\text { Relato de caso } \\
\text { (Manger, Streitberg, } \\
\text { Seitz, Kleyer \& } \\
\text { Manger, 2017) }\end{array}$ & $50 / \mathrm{F}$ & Palato duro & $\begin{array}{l}\text { Mancha cinza-azulada } \\
\text { indolor }\end{array}$ & $\begin{array}{l}\text { Cloroquina } 250 \\
\mathrm{mg} / \text { dia / } 4 \text { anos }\end{array}$ & $\begin{array}{l}\text { Síndrome de } \\
\text { Sjögren }\end{array}$ \\
\hline $\begin{array}{l}\text { Relato de caso } \\
\text { (de Andrade et al., } \\
\text { 2017) }\end{array}$ & $60 / \mathrm{F}$ & Palato duro & $\begin{array}{c}\text { Mancha azul-acinzentada } \\
\text { difusa }\end{array}$ & $\begin{array}{l}\text { Cloroquina } 150 \\
\mathrm{mg} / \text { dia / } 1 \text { ano }\end{array}$ & $\begin{array}{l}\text { Artrite } \\
\text { reumatoide }\end{array}$ \\
\hline $\begin{array}{l}\text { Relato de caso } \\
\text { (de Melo Filo et al., } \\
\text { 2012) }\end{array}$ & $64 / F$ & $\begin{array}{l}\text { Palato duro, } \\
\text { dorso de língua, } \\
\text { gengiva inferior }\end{array}$ & $\begin{array}{l}\text { Manchas escuras difusas e } \\
\text { com bordas irregulares }\end{array}$ & $\begin{array}{l}\text { Cloroquina } 100 \\
\text { mg/dia / } 15 \text { anos }\end{array}$ & $\begin{array}{l}\text { Artrite } \\
\text { reumatoide }\end{array}$ \\
\hline
\end{tabular}

Fonte: Elaborado pelo autor (2021).

\section{Discussão}

Os antimaláricos são um dos medicamentos mais conhecidos por apresentarem pigmentação da mucosa oral como efeito adverso naqueles indivíduos que necessitam de uso contínuo (Ferrazzo, Payeras, Surkamp \& Danesi, 2017). A pigmentação da mucosa oral pode ter diversas etiologias e envolver uma variedade imensa de lesões e condições. Para isso é necessário uma anamnese completa e detalhada, bem como um estudo profundo do caso clínico através do exame físico, dessa forma será possível realizar o diagnóstico diferencial adequado (Gadelha, Moura, Medeiros, Melo \& Vasconcelos, 2014). 
Dos 18 casos analisados, nota-se que 15 se apresentaram no palato duro, sendo esse o local de maior acometimento. Ferrazzo e colaboradores (2017) afirmaram que a pigmentação por medicamento antimalária (cloroquina) na mucosa do palato foi relatada primeiramente em 1945 por soldados do Pacífico Sul e desde então vários estudos foram realizados e descritos. A grande parte dos casos envolvia apenas o palato duro havendo ainda uma linha que demarcava junção com o palato mole. Porém a literatura não deixa explícito o real motivo para o envolvimento exclusivo do palato duro (Horta-Baas, 2018).

A pigmentação da mucosa oral, de acordo com o caso clínico publicado por Ferrazzo e colaboradores (2017), são mudanças que evoluem desde máculas isoladas para grandes lesões em lábios e mais comumente em palato. São geralmente assintomáticas, de cor azul e cinza, com demarcação notável entre o palato mole e o duro. E quando induzidas por drogas são mais enegrecidas e exibem bordas irregulares, cujo aspecto é semelhante a lesões malignas como os melanomas, enfatizando a importância do diagnóstico diferencial.

As áreas pigmentadas geralmente são coloridas envolvendo os tons de cinza-azulado ou preto-azulado, com uma apresentação de tamanho variável e podem estar bem circunscritas (de Andrade et al., 2013; Giansanti, Tillery \& Olansky, 1971; Horta-Baas, 2018). Dos relatos de casos analisados a variação de cor das lesões não foi considerável, pois a grande parte estava dentro da tonalidade azulada, estando de acordo com as informações presentes na literatura.

Em um estudo dirigido por M. A. Melikoglu, M. Melikoglu, Gurbuz, Budak e Kacar (2008), 25\% dos pacientes recebendo antimaláricos como Cloroquina, quinacrina ou amodiaquina por pelo menos 4 meses desenvolveram hiperpigmentação. O que corrobora com os resultados dessa pesquisa, pois dos relatos estudados, as primeiras aparições das lesões se deram com 4 meses de uso do medicamento. No entanto, a hiperpigmentação ao consumo de hidroxicloroquina é rara e há muito poucos casos publicados, possivelmente porque ela contém dois terços do efeito da Cloroquina (Kasper, Flueckiger, Gobbi, Lautenschlager \& Borelli, 2014; Ochsendorf, 2010).

A pigmentação pelo uso de cloroquina pode ser um indicador de envolvimento ocular, acarretando o desenvolvimento de retinopatias, que muitas vezes pode ser irreversível e, em casos mais graves, leva à cegueira (Gallo et al., 2009; Stewart, Miles \& Earnshaw, 1968). A dose diária de cloroquina parece ser relevante para o desenvolvimento da ocular doença e deve ser estabelecida dependendo do peso do paciente, não superior a 4mg / kg / dia (Lerman, Karimbux, Guze \& Woo, 2009). Marmor, Carr, Easterbrook, Farjo e Mieler (2002) demonstraram no caso de terapia com cloroquina, que o risco de retinopatia é mínima, quando a dose diária não excede $250 \mathrm{mg}$ (Andrade et al., 2019). Por isso, todo paciente em uso de cloroquina e seus derivados devem ser acompanhados e documentados desde seu início(Lacava, 2010). A equipe odontológica deve estar sempre atenta aos sinais e sintomas, para que o diagnóstico precoce seja realizado, assim como os encaminhamentos necessários para outras especialidades.

Alguns autores relatam que o efeito adverso da hiperpigmentação associada ao consumo de hidroxicloroquina é improvável em doses inferiores ao máximo recomendado $6,5 \mathrm{mg} / \mathrm{kg}$ de peso ideal por dia, outros mencionam que não há preditor conhecido de quem desenvolverá hiperpigmentação (Andrade et al., 2019; Dereure, 2001).

As manifestações clínicas de pigmentações devido ao uso de medicamentos podem variar, mas grande parte produz uma melanose difusa, já outras podem causar um padrão único. As mulheres mostram-se mais sensíveis ao aumento da pigmentação por melanina, provavelmente em decorrência da interação com os hormônios sexuais (da Silveira et al., 2003; Neville, Damm, Allen \& Bouquot, 1998). O que torna relevante e justifica o fato de que dos 18 casos deste estudo, 16 pertenciam ao sexo feminino.

Ao longo da história tem sido atribuído a capacidade antiviral da cloroquina e hidroxicloroquina, sendo capazes de inibir o crescimento dos vírus. Atualmente com a pandemia pelo COVID-19 decidiu-se investigar a utilidades desses medicamentos com base em antecedentes (Abuabara-Franco et al., 2020). Existem várias evidências pré-clínicas e levantamento da eficácia dos antimaláricos contra o COVID-19. Com base em resultados de estudos preliminares, autoridades 
de saúde de diversos países têm recomendado indiscriminadamente o uso no tratamento desse novo vírus (Pacheco, Pachito, Bagattini \& Riera, 2000).

Porém, na tentativa de tentar controlar a pandemia e na busca rápida por medicamentos para o tratamento desse vírus, no presente momento não existe embasamento científico para o uso de antimaláricos nos casos de COVID-2019. Embora exista evidências sólidas do uso desses medicamentos apenas em outras situações médicas, o seu uso continua sendo uma recomendação "off label" e com base em medicina translacional (Neville et al., 1998). No entanto, como mencionado por Pacheco e colaboradores (2020) o embasamento para uso de medicamentos para casos de COVID-19, assim como para qualquer outra doença, deve ser pautado na existência de estudos clínicos de qualidade e não devem ser justificados unicamente por seus potenciais mecanismos de ação observados em estudos experimentais pré-clínicos.

Quanto às pigmentações, não há necessidade de tratamento para essas lesões, embora possa haver preocupações estéticas. Há relatos de uma melhoria considerável quando se retira o agente infrator, mas as pigmentações induzidas por drogas podem permanecer por diversos anos (Gallo et al., 2009; Kalia \& Dutz, 2007).

\section{Considerações Finais}

De acordo com os estudos analisados na literatura, a região da mucosa oral mais afetada pelas pigmentações causadas pelo uso dos antimaláricos foi o palato duro, apresentando na maioria das vezes uma cor azul-acinzentada e acometendo principalmente mulheres. Além disso, o uso da Cloroquina foi prevalente ao da Hidroxicloroquina na maioria dos casos.

Diante disso, espera-se que este estudo ajude os cirurgiões-dentistas no correto diagnóstico das pigmentações na cavidade oral, atentando-se, dentre outras coisas, sobre a importância de uma correta anamnese para averiguar o possível uso desses medicamentos, o tempo e a quantidade. Além disso, é importante também que o profissional saiba fazer o correto diagnóstico diferencial com as outras pigmentações que podem acometer a cavidade oral.

Infere-se, ainda, a necessidade de realização de estudos mais aprofundados sobre a temática abordada a fim de fornecer o suporte adequado quanto as consequências do uso indevido da Cloroquina e Hidroxicloroquina, no intuito de elucidar seus efeitos na cavidade oral, tendo em vista também o aumento exacerbado na utilização desses medicamentos por parte da população, frequentemente sem orientação médica, como terapia suporte para a COVID-19.

\section{Referências}

Abuabara-Franco, E., Bohórquez-Rivero, J., Restom-Arrieta, J., Sáenz-López, J., Correa-Guerrero, J., \& Mendoza-Paternina, C. (2020). Consideraciones actuales de antimaláricos en la infección por SARS-COV-2 y su impacto. Revista Colombiana de Nefrología, 7(Supl. 2).

Andrade, L. G. M., Rojas, D. O. P., Rodríguez, G. R., \& Vázquez, F. L. (2019). Hiperpigmentación mucocutánea inducida por hidroxicloroquina en un paciente con lupus eritematoso sistémico. Dermatología Cosmética, Médica y Quirúrgica, 17(2), 111-114.

Attuati, S., Martini, V., Bonacina, R., Mariani, U., \& Gianatti, A. (2019). Hard Palate Hyperpigmentation Induced by Chloroquine: A Case Report. Multidisciplinary Digital Publishing Institute Proceedings, 35(1), 45.

da Silveira, H. E. D., de Quadros, O. F., Maito, F. L. D. M., \& Giordani, S. (2003). Hiperpigmentação da mucosa bucal causada pelo uso prolongado de fármaco antimalárico. Revista da Faculdade de Odontologia de Porto Alegre, 44(1), 36-38.

de Andrade, B. A. B., Fonseca, F. P., Pires, F. R., Mesquita, A. T. M., Falci, S. G. M., dos Santos Silva, A. R., \& de Almeida, O. P. (2013). Hard palate hyperpigmentation secondary to chronic chloroquine therapy: report of five cases. Journal of cutaneous pathology, 40(9), 833-838.

de Andrade, B. A. B., Padron-Alvarado, N. A., Muñoz-Campos, E. M., Morais, T. L., \& Martinez-Pedraza, R. (2017). Hyperpigmentation of hard palate induced by chloroquine therapy. Journal of clinical and experimental dentistry, 9(12), e1487.

de Melo Filho, M. R., da Silva, C. A. D., da Rocha Dourado, M., de Oliveira Pires, M. B., Pêgo, S. P. B., \& de Freitas, E. M. (2012). Palate hyperpigmentation caused by prolonged use of the anti-malarial chloroquine. Head and neck pathology, 6(1), 48-50.

Dereure, O. (2001). Drug-induced skin pigmentation. American journal of clinical dermatology, 2(4), 253-262.

Dong, L., Hu, S., \& Gao, J. (2020). Discovering drugs to treat coronavirus disease 2019 (COVID-19). Drug discoveries \& therapeutics, 14(1), 58-60. 
Ferrazzo, K. L., Payeras, M. R., Surkamp, P., \& Danesi, C. C. (2017). Pathological pigmentation of the skin and palate caused by continuous use of chloroquine: case report. Journal of Oral Diagnosis, 2(1), 1-5.

Gallo, C. B., Luiz, A. C., Ferrazzo, K. L., Migliari, D. A., \& Sugaya, N. N. (2009). Drug-induced pigmentation of hard palate and skin due to chronic chloroquine therapy: report of two cases. Clinical and experimental dermatology, 34(7).

Gao, J., Tian, Z., \& Yang, X. (2020). Breakthrough: Chloroquine phosphate has shown apparent efficacy in treatment of COVID-19 associated pneumonia in clinical studies. Bioscience trends.

Giansanti, J. S., Tillery, D. E., \& Olansky, S. (1971). Oral mucosal pigmentation resulting from antimalarial therapy. Oral Surgery, Oral Medicine, Oral Pathology, 31(1), 66-69.

Godinho, G. V., Paz, A. L. L. M., de Araújo Gomes, E. P. A., Garcia, C. L., \& Volpato, L. E. R. (2020). Extensive hard palate hyperpigmentation associated with chloroquine use. British journal of clinical pharmacology, 86(11), 2325-2327.

Gulati, N., Dutt, P., Gupta, N., \& Tyagi, P. (2016). Gingival pigmentation: revisited. Journal of Advanced Medical and Dental Sciences Research, 4(1), 48.

Holmstrup, P., Plemons, J., \& Meyle, J. (2018). Non-plaque-induced gingival diseases. Journal of clinical periodontology, 45, S28-S43.

Horta-Baas, G. (2017). Chloroquine-induced oral mucosal hyperpigmentation and nail dyschromia. Reumatología clinica, 14(3), 177-178.

Kalampalikis, A., Goetze, S., \& Elsner, P. (2012). Isolated hyperpigmentation of the oral mucosa due to hydroxychloroquine. Journal der Deutschen Dermatologischen Gesellschaft = Journal of the German Society of Dermatology: JDDG, 10(12), 921-922.

Kalia, S., \& Dutz, J. P. (2007). New concepts in antimalarial use and mode of action in dermatology. Dermatologic therapy, 20(4), 160-174.

Kasper, R. S., Flueckiger, B., Gobbi, S., Lautenschlager, S., \& Borelli, S. (2014). Hydroxychloroquine-induced hyperpigmentation. Journal of the German Society of Dermatology: JDDG, 12(2), 158-161.

Lacava, A. C. (2010). Complicações oculares da terapêutica com a cloroquina e derivados. Arquivos Brasileiros de Oftalmologia, 73(4), 384-389.

Lerman, M. A., Karimbux, N., Guze, K. A., Woo, S. B. (2009). Pigmentation of the hard palate. Oral Surgery, Oral Medicine, Oral Pathology, and Oral Radiology, 107(1), 8-12.

Marmor, M. F., Carr, R. E., Easterbrook, M., Farjo, A. A., \& Mieler, W. F. (2002). Recommendations on screening for chloroquine and hydroxychloroquine retinopathy: a report by the American Academy of Ophthalmology. Ophthalmology, 109(7), 1377-1382.

Melikoglu, M. A., Melikoglu, M., Gurbuz, U., Budak, B. S., \& Kacar, C. (2008). Hydroxychloroquine-induced hyperpigmentation: a case report. Journal of clinical pharmacy and therapeutics, 33(6), 699-701.

Neville, B. W., Damm, D. D., Allen, C. M., \& Bouquot, J. E. (1998). Patologia oral e maxillofacial (1a ed.): Guanabara Koogan.

Ochsendorf, F. (2010). Use of antimalarials in dermatology. Journal of the German Society of Dermatology: JDDG, 8(10), 829-44.

Pacheco, R. L., Pachito, D. V., Bagattini, A. M, Riera, R. (2020). Hidroxicloroquina E Cloroquina Para COVID-19. Revisão sistemática rápida. https://docs.bvsalud.org/biblioref/2020/06/1099475/rs_rapida_hidroxicloroquina_covid19_atualizacao_21_05_20.pdf.

Pereira, A. S., Shitsuka, D. M., Parreira, F. J., \& Shitsuka, R. (2018). Metodologia da pesquisa científica. [e-book].

Rodrigo, R. G., Ilnara, I. S. M., Layssa, L. K. D. S. M., de Melo Daniel, D. S., \& Marcelo, M. G. V. (2014). As principais lesões enegrecidas da cavidade oral. Rev Cubana Estomatol, 51(2), 39-44.

Shahna, N., Suchetha, A., Sapna, N. D. D. B., \& Apoorva, S. M. (2019). Gingival pigmentation: A review of literature. Int J Appl Dent Sci, 5(2), 83-91.

Shuai, Y., Wang, J., Jiang, H., Yu, Y., \& Jin, L. (2020). Oral-maxillofacial adverse events related to antimalarials. Oral Diseases.

Sreeja, C., Ramakrishnan, K., Vijayalakshmi, D., Devi, M., Aesha, I., \& Vijayabanu, B. (2015). Oral pigmentation: a review. Journal of pharmacy \& bioallied sciences, 7(Suppl 2), S403.

Steffens, J. P., \& Marcantonio, R. A. C. (2018). Classificação das doenças e condições periodontais e peri-implantares 2018: guia prático e pontos-chave. Revista de Odontologia da UNESP, 47(4), 189-197.

Stewart, T. W., Miles, D. W., Earnshaw, E. R. (1968). Pigmentation and retinopathy due to chloroquine. Acta Dermato-Venereologica, 48(1), 47-52.

Tosios, K. I., Kalogirou, E. M., \& Sklavounou, A. (2018). Drug-associated hyperpigmentation of the oral mucosa: report of four cases. Oral surgery, oral medicine, oral pathology and oral radiology, 125(3), e54-e66.

Wang, M., Cao, R., Zhang, L., Yang, X., Liu, J., Xu, M., \& Xiao, G. (2020). Remdesivir and chloroquine effectively inhibit the recently emerged novel coronavirus (2019-nCoV) in vitro. Cell research, 30(3), 269-271.

Yao, X., Ye, F., Zhang, M., Cui, C., Huang, B., Niu, P., \& Liu, D. (2020). In vitro antiviral activity and projection of optimized dosing design of hydroxychloroquine for the treatment of severe acute respiratory syndrome coronavirus 2 (SARS-CoV-2). Clinical infectious diseases, 71(15), 732-739. 